\title{
Discovery of a cataclysmic variable with a sub-stellar companion
}

\author{
R. E. Mennickent ${ }^{1, \star}$, M. Diaz ${ }^{2}$, W. Skidmore ${ }^{3}$, and C. Sterken ${ }^{4}$ \\ 1 Departamento de Fisica, Universidad de Concepcion, Casilla 160, Concepcion, Chile \\ 2 Instituto Astronomico e Geofisico, Universidade de Sao Paulo, Brazil \\ 3 School of Physics and Astronomy, University of St. Andrews, North Haugh, St. Andrews, Fife, KY16 9SS, UK \\ ${ }^{4}$ University of Brussels (VUB), Pleinlaan 2, 1050 Brussels, Belgium
}

Received 15 January 2001 / Accepted 13 June 2001

\begin{abstract}
We find that the ROSAT source 1RXS J105010.3-140431 is a cataclysmic variable with orbital period of 88.6 min and a spectrum closely resembling WZ Sge. In particular, emission lines are flanked by Stark-broadened absorption wings probably originating in the photosphere of a compact object. The Balmer absorption lines can be modeled by the spectrum of a DA white dwarf with $13000<T_{\text {eff }}<24000 \mathrm{~K}$. The strong absorption lines allowed us to obtain direct radial velocities of the white dwarf using the cross-correlation technique. We find an extremely low white dwarf radial velocity half amplitude, $K_{\mathrm{wd}}=4 \pm 1 \mathrm{~km} \mathrm{~s}^{-1}$. This is consistent with the upper limit obtained from the $\mathrm{H} \alpha$ emission line wing $K<20 \mathrm{~km} \mathrm{~s}^{-1}$. The corresponding mass function is incompatible with a main sequence secondary, but is compatible with a post orbital period minimum cataclysmic variable with a brown dwarf-like secondary. The formal solution gives a secondary mass of 10-20 Jovian masses. Doppler maps for the emission lines and the hypothesis of black-body emission indicate a steady state $\left(T \sim r^{-3 / 4}\right)$ accretion disk mainly emitting in $\mathrm{H} \alpha$ and an optically thicker hotspot with a strong contribution to the higher order Balmer lines and He I 5875. As in other long cycle length dwarf novae, evidence for inner disk removal is found from the analysis of the emission lines.
\end{abstract}

Key words. accretion, accretion disks - stars: binaries: close - stars: individual: 1RXS J105010.3-140431 stars: novae, cataclysmic variables

\section{Introduction}

1RXS J105010.3-140431 $\left(\alpha_{2000}=+105010.4, \delta_{2000}=\right.$ $-140432.0)$ is a poorly-studied ROSAT source detected with a count rate of $0.06018 \pm 0.01495$ counts $/ \mathrm{s}$ (Voges et al. 1996). In this paper we present the first spectroscopic and photometric study of this object, revealing a probable classification as a dwarf nova in the very late stages of the evolutionary track of cataclysmic variables $(\mathrm{CVs})$.

\section{Observations and data reduction}

\subsection{Photometric observations}

We observed 1RXS J105010.3-140431 during three nights on February 17, 19 and 20, 2000 with the Danish-1.54 m telescope of the ESO La Silla Observatory. The Danish Faint Object Spectrograph and Camera DFOSC was used with the backside illuminated Loral/Lesser chip. This CCD has $2052 \times 205215 \mu \mathrm{m}$ pixels spanning a field of

\footnotetext{
Send offprint requests to: $\mathrm{R}$. E. Mennickent,

e-mail: rmennick@stars.cfm.udec.cl

* Based on observations obtained at the European Southern Observatory, ESO proposals 58.D-0349 and 64.H-0065(B).
}

view of $13.3 \times 13^{\prime} .3$. A windowing of $5^{\prime} \times 5^{\prime}$ was selected around the center to ensure fast readout of the CCD. We imaged the field of the variable continuously for approximately $\sim 2$ hours every night with the $V$ filter and then measured the instrumental magnitudes of the variable and comparison stars using the aperture photometry facility in the software MIRA AP ${ }^{1}$. Differential photometry was performed using nearby isolated stars in the field. This technique is robust against small changes in atmospheric transparency and today it is routinely used for monitoring variable stars (i.e. Howell et al. 1988). A finding chart indicating the comparison stars is shown in Fig. 1. The $\mathrm{C} 2-\mathrm{C} 1$ light curve had a mean value of $-1.04 \mathrm{mag}$ and rms 0.017 mag whereas the $\mathrm{C} 3-\mathrm{C} 2$ light curve showed an average of $1.45 \mathrm{mag}$ and $\mathrm{rms} 0.020 \mathrm{mag}$. We did not use the bright star close to the variable as a comparison since it was saturated in our frames. As C2 has a magnitude similar to the variable, we deduce an upper limit error of $0.017 \mathrm{mag}$ for the $\mathrm{V}-\mathrm{C} 2$ curve. This light curve, along with the errors, are shown in Fig. 2, suggesting that the star is marginally variable every night at the $2 \sigma$ level,

${ }^{1}$ MIRA is a registered trademark of Axiom Research, Inc. 


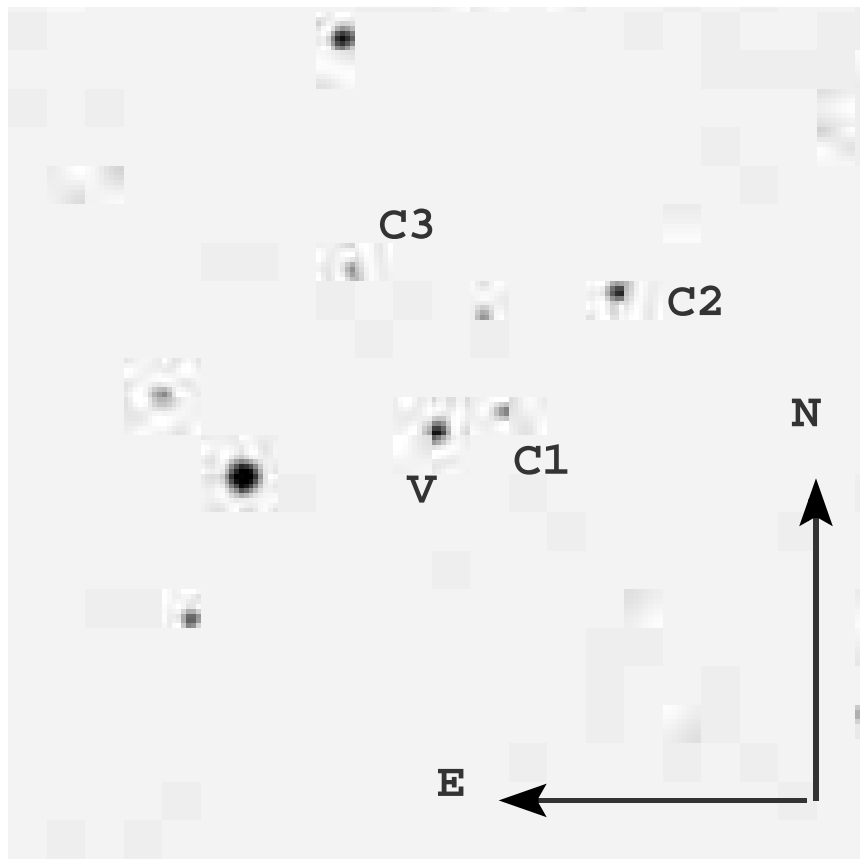

Fig. 1. An ESO Digitised Sky Survey image indicating the comparison stars used for differential photometry. The field of view is $5^{\prime} \times 5^{\prime}$.

but the mean magnitude changes from night to night by 0.05 mag.

\subsection{Spectroscopic observations}

Spectra were obtained on the 2.2 meter telescope at ESO's La Silla Observatory, on January 4-7, 1997. The EFOSC2 and CCD \# 24, along with grism \# 4 and a slit width of 1 arcsec, yielded a spectral resolution of $3.4 \AA$, as determined by the slit width, plate scale and pixel size, and a wavelength range of 4020-7470 ̊. He-Ar comparison spectra were taken every five 480-s length science exposures. Bias and flat images were taken daily. Observations are summarized in Table 1.

Data reduction was performed in the usual manner using the IRAF software package ${ }^{2}$. All of the two dimensional raw frames were de-biased and flat-fielded using the average bias and normalized flat images, and then one dimensional spectra were extracted. About $30 \mathrm{He}-\mathrm{Ar}$ emission lines provided spectral calibration functions with typical rms of $0.4 \AA\left(18 \mathrm{~km} \mathrm{~s}^{-1}\right.$ at $\left.\mathrm{H} \alpha\right)$. Flux calibration was performed with the standard stars LTT 1020 and EG 21 (Hamuy et al. 1992; Hamuy et al. 1994).

\section{The analysis of the emission line spectrum}

The top of Fig. 3 shows the averaged spectrum of the object, characterized by $\mathrm{H}$ I lines and He I emission. Flanking

\footnotetext{
${ }^{2}$ IRAF is distributed by the National Optical Astronomy Observatories, which are operated by the Association of Universities for Research in Astronomy, Inc., under cooperative agreement with the National Science Foundation.
}

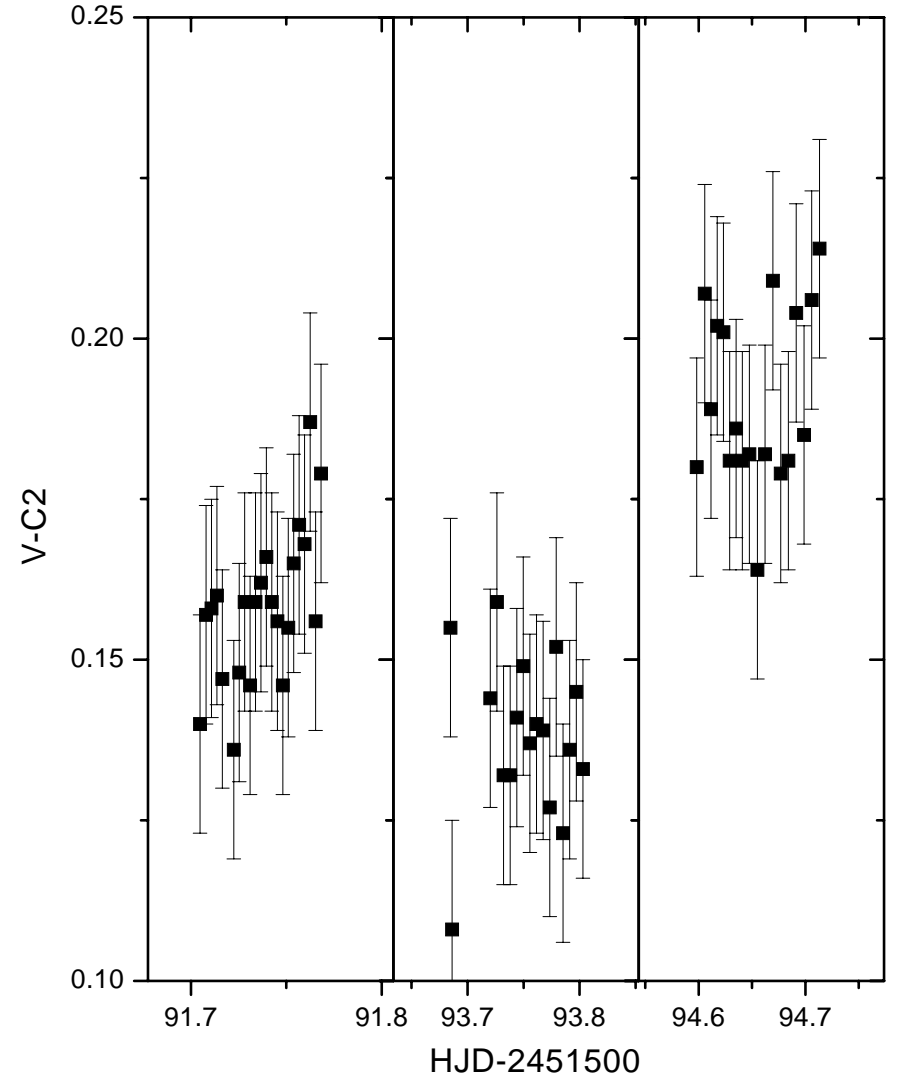

Fig. 2. The differential magnitudes in the $V$ band along with their error bars.

Table 1. The spectroscopic observing log indicating starting and ending times for the time series and nightly mean $V$ magnitude. The number of science frames per night is also given.

\begin{tabular}{|c|c|c|c|c|}
\hline \hline Date(UT) & \multicolumn{1}{c}{$N$} & HJD $_{\text {start }}$ & \multicolumn{1}{c}{ HJD $_{\text {end }}$} & $V$ \\
\hline $05 / 01 / 97$ & 13 & 53.7688 & 53.8676 & $17.63(09)$ \\
$06 / 01 / 97$ & 14 & 54.7928 & 54.8188 & $17.82(10)$ \\
$07 / 01 / 97$ & 14 & 55.7700 & 55.8647 & $17.65(05)$ \\
$08 / 01 / 97$ & 17 & 56.7590 & 56.8704 & $17.48(16)$ \\
\hline \hline
\end{tabular}

the strong Balmer emission lines we observe the pressure broadened absorption profiles typical of a white dwarf. We are confident that these lines come from a white dwarf and not from a hot disk, since the helium lines are not observed in absorption, like in outbursting dwarf novae (for a review of spectra during outbursts see Warner 1995, see also Sect. 4). The $\mathrm{H} \alpha$ emission is double peaked (see bottom panel of Fig. 3) with an equivalent width of $-110 \AA$ and half peak separation $560 \mathrm{~km} \mathrm{~s}^{-1}$. The full width at half maximum $F W H M$ in $\mathrm{H} \alpha$ is $1811 \mathrm{~km} \mathrm{~s}^{-1}$ and the full width at zero intensity $F W Z I$ is $2950 \mathrm{~km} \mathrm{~s}^{-1}$. These values are typical for dwarf nova accretion disks at quiescence. Spectroscopic quantities for the averaged spectrum are given in Table 2. The steep emission decrement suggests an origin in an optically thin accretion disk. Although no outburst has been observed yet for this object, it is likely that it corresponds to a dwarf nova of the SU UMa subtype. These systems, reviewed by Warner (1995, 2000), consist of a white dwarf accreting 

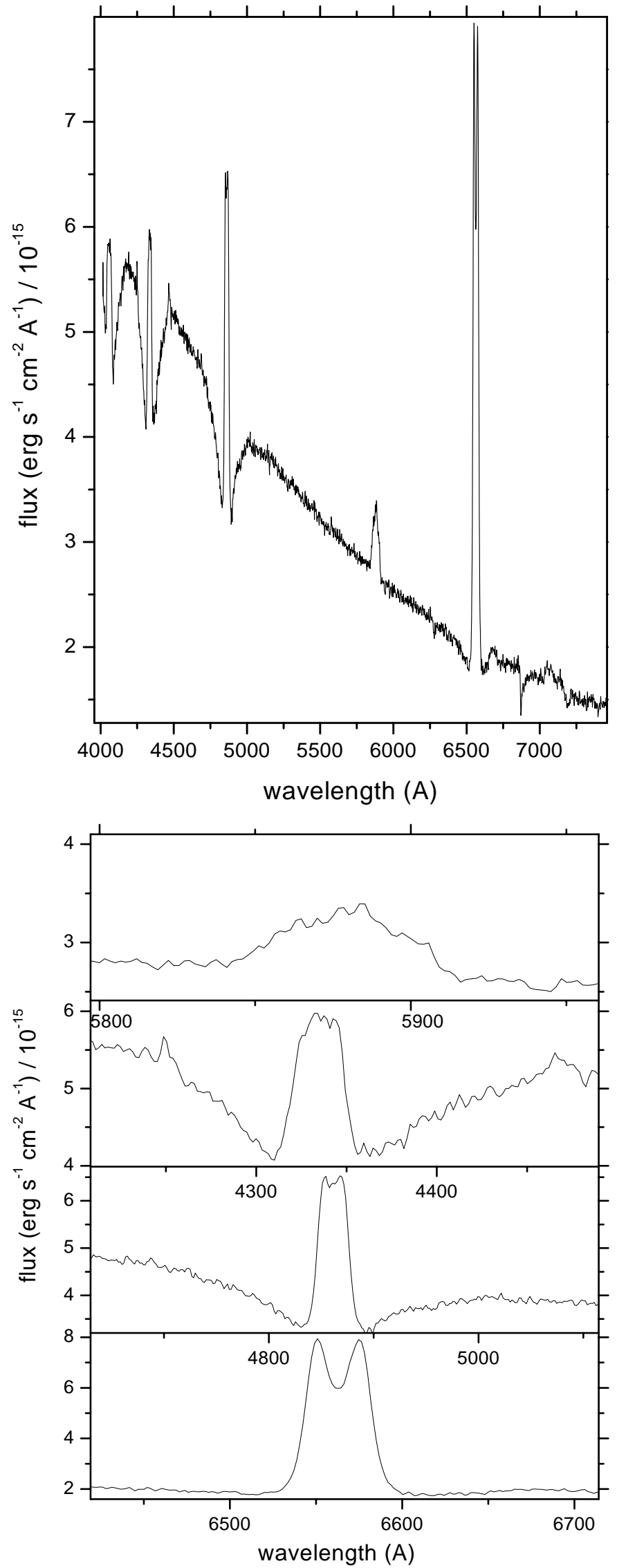

Fig. 3. The averaged spectrum (upper panel) and a close up view of the main spectral lines (below panel). From up to down the $\mathrm{He} 5875, \mathrm{H} \gamma, \mathrm{H} \beta$ and $\mathrm{H} \alpha$ lines are shown.
Table 2. Mean spectroscopic quantities. Positive equivalent widths correspond to the absorption component, excluding the emission core.

\begin{tabular}{|c|c|c|}
\hline \hline \multicolumn{1}{c}{ Line } & \multicolumn{1}{c}{ flux $\left(\mathrm{erg} \mathrm{cm}^{-2} \mathrm{~s}^{-1} \AA^{-1}\right)$} & $W_{\lambda}(\AA)$ \\
$\mathrm{H} \delta$ & $5.9 \mathrm{E}-16$ & -8 \\
$\mathrm{H} \gamma$ & $6.0 \mathrm{E}-16$ & $-13 /+33$ \\
$\mathrm{He} \mathrm{I} 4470$ & $5.5 \mathrm{E}-16$ & -1 \\
$\mathrm{H} \beta$ & $6.5 \mathrm{E}-16$ & $-33 /+38$ \\
$\mathrm{He} \mathrm{I} 5875$ & $3.4 \mathrm{E}-16$ & -10 \\
$\mathrm{H} \alpha$ & $7.9 \mathrm{E}-16$ & -129 \\
$\mathrm{He}$ I 6678 & $2.0 \mathrm{E}-16$ & -2 \\
\hline \hline
\end{tabular}

material from a cool red dwarf at very low transfer rates, producing thermal instabilities (outbursts) in an accretion disk with recurrence times from weeks to several years. The faint continuum produced in the disk is hidden in the brighter white dwarf spectrum even in optical wavelengths, producing the absorption wings seen around the Balmer emission cores. In this regard the spectrum is quite similar to that of the dwarf nova GW Lib (Szkody et al. 2000).

\subsection{The orbital period and ephemeris}

Derivation of stellar parameters for the binary components in a dwarf nova requires a precise determination of the radial-velocity curve of at least one of the stars. This task is not always possible, because in most systems the white dwarf and secondary star spectrum is hidden in the optical spectrum of the bright accretion disk. In fact, the emission lines originating in the disk usually do not reflect the white dwarf binary motion because they are subject to intrinsic velocity fields besides the gas rotation in the disk itself (e.g. Robinson 1992). This yields an intrinsic uncertainty in the spectroscopic ephemeris obtained for most dwarf novae. The problem has been overcome in long orbital period dwarf novae by observing in the infrared the lines of the secondary star. In these systems the relative flux contribution and features of the secondary star allow a straightforward measurement (e.g. Friend et al. 1990). However, short orbital period dwarf novae have secondaries too faint to be detected even in the IR with current instrumentation. In addition, it is difficult to derive precise radial velocities (RV) using molecular bandprofiles. Fortunately, the disk in 1RXS J105010.3-140431 is faint enough to reveal the white dwarf photospheric lines, so we have a unique opportunity to obtain white dwarf radial velocities and the unbiased ephemeris for the system. The spectra were normalized by dividing them with a loworder polynomial continuum fit before we performed the radial velocity analysis.

We traced the motion of the $\mathrm{H} \beta$ and $\mathrm{H} \gamma$ emission line peak by measuring their radial velocity. Then we constructed periodograms using the $A O V$ algorithm (Schwarzenberg-Czerny 1989) and the Scargle (1982) method. We scanned a frequency range between $90 \mathrm{c} / \mathrm{d}$ 


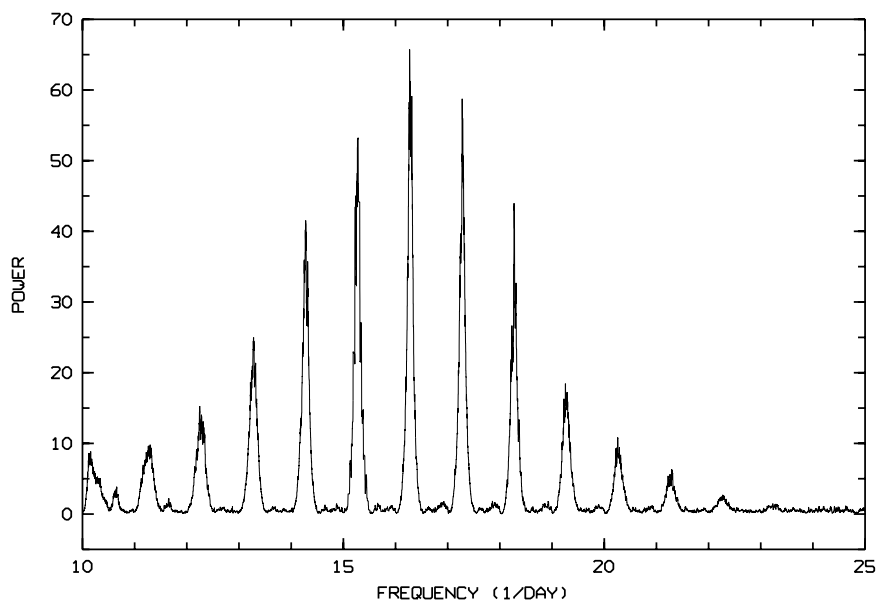

Fig. 4. The $A O V$ periodogram of the $\mathrm{H} \beta$ emission line. The maximum at $16.27 \mathrm{c} / \mathrm{d}(0.0615)$ is identified as the orbital period.

(the Nyquist frequency) and $1 \mathrm{c} / \mathrm{d}$. Both methods yielded consistent results.

The $A O V$ periodogram is shown in Fig. 4. The main peak is found at $16.272 \pm 0.058 \mathrm{c} / \mathrm{d}$, along with $\pm 1 \mathrm{c} / \mathrm{d}$ aliases at 15.3 and $17.3 \mathrm{c} / \mathrm{d}$. Based on the strength of the main maximum and assuming a power window symmetrical regarding the absolute minimum, we selected 16.272 $\mathrm{c} / \mathrm{d}(0 \mathrm{~d} 0615 \pm 0 \mathrm{~d} 0002)$ as the most likely period, corresponding probably to the binary orbital period. Due to the possible biased nature of the emission line radial velocities, we used the $\mathrm{H} \gamma$ absorption line (see next section) as the best tracer of the white dwarf binary motion, measuring the half amplitude of their radial velocity which resulted basically independent of the period alias, giving confidence about the stellar parameters derived in Sect. 3.5. We found the following ephemeris for the inferior conjunction of the secondary star:

$\mathrm{HJD}=2450453.71479(308)+0.0615(2) E$.

\subsection{Comparison between emission and absorption radial velocities}

In this section we compare the radial velocity sets of emission and absorption components obtained with different methods. It was extremely difficult to find reliable velocities for the absorption component. First we tried extracting the emission core and then fitting a Gaussian function to the wings, but the line center was poorly constrained and we obtained noisy results. Secondly we tried deblending the spectral line with emission plus absorption Gaussians - using the "d" key in the splot IRAF package, obtaining a large amplitude for the absorption component (around $110 \mathrm{~km} \mathrm{~s}^{-1}$ ). However, numerical simulations showed that this result was an artifact: the same apparent oscillation was obtained with a synthetic zero velocity absorption profile and a moving - $600 \mathrm{~km} \mathrm{~s}^{-1}$ amplitude - emission component (the $s$-wave, see below). Finally, we applied the cross-correlation technique

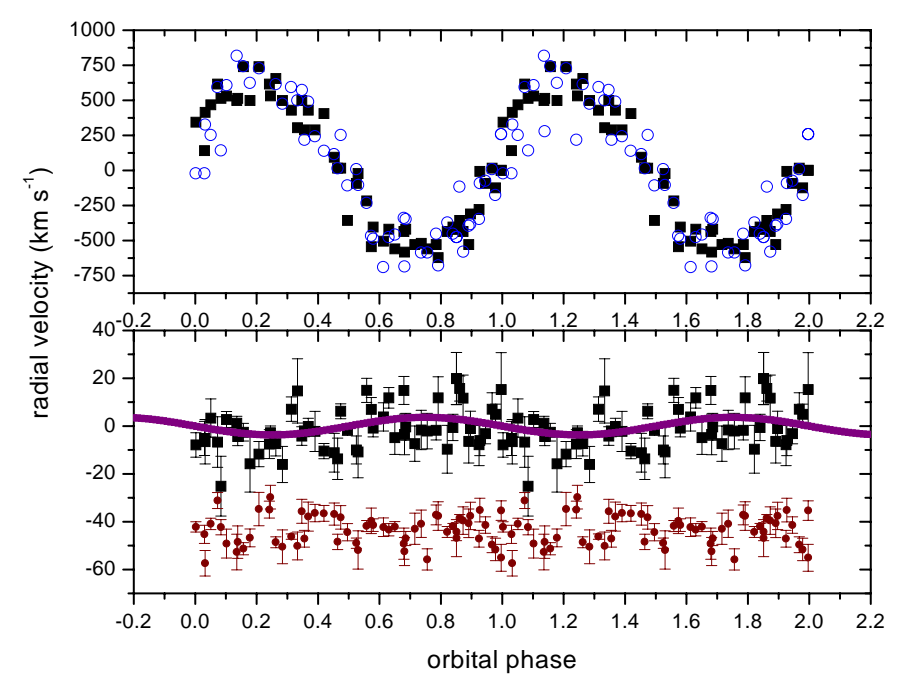

Fig. 5. Upper panel: the $\mathrm{H} \beta$ (squares) and $\mathrm{H} \gamma$ (circles) $s$-wave velocity folded with the ephemeris given by Eq. (1). Below panel: the $\mathrm{H} \gamma$ absorption line velocity (squares) obtained by cross correlation and the best sine fit along with results for zero velocity synthetic absorption profiles (circles).

(Tonry \& Davis 1979), implemented in the $f x c$ IRAF task, to the $\mathrm{H} \beta$ and $\mathrm{H} \gamma$ white dwarf absorption, excluding the emission component inside a range of $-2500 \mathrm{~km} \mathrm{~s}^{-1}<v<$ $2500 \mathrm{~km} \mathrm{~s}^{-1}$. Results for $\mathrm{H} \beta$ turned to be too noisy, but the $\mathrm{H} \gamma$ velocity, binned with the orbital period and fitted with a sine function, allowed us to calculate the time of inferior conjunction of the secondary star, $T_{0}$ and the radial velocity half-amplitude: $K_{\mathrm{wd}}=4 \pm 1 \mathrm{~km} \mathrm{~s}^{-1}$ (Fig. 5 ).

In Fig. 5 we observe the contrast between the large amplitude of the emission line peak and the white dwarf velocity. We will show that the large amplitude of the emission peak $\left(619 \pm 19 \mathrm{~km} \mathrm{~s}^{-1}\right.$ for $\left.\mathrm{H} \beta\right)$ reflects the characteristic $s$-wave observed in the trailed spectra of some dwarf novae in quiescence. Note that this $s$-wave moves in antiphase with the white dwarf, consistent with an origin close to the inner lagrangian point.

Figure 5 also shows that the low $K$ amplitude is inconsistent with noise, as inferred from measurements made on synthetic spectra with zero velocity and noise similar to the original dataset. We explored the possibility that the low velocity pattern in Fig. 5 is the effect of residual high velocity emission. However, the $s$-wave phasing and the disk emission phasing (see below) indicate that, if present, it should increase the measured amplitude if coming from the $s$-wave and should have minor effects if coming from the disk (the effects are summed or canceled according to the relative phasing).

The inferred $K_{\text {wd }}$ value is amazing. Their accuracy probably lies in the statistical significance of the crosscorrelation technique, which fits a large sample of pixels simultaneously. Interestingly, low $K_{\mathrm{wd}}$ values have also been observed in other low mass-transfer rate dwarf nova which reveal the white dwarf in their optical spectra, namely WZ Sge (40 $\pm 10 \mathrm{~km} \mathrm{~s}^{-1}$, Spruit \& Rutten 1998), GW Lib (40 $\mathrm{km} \mathrm{s}^{-1}$, Szkody et al. 2000) and AL Com 
$\left(0 \pm 32 \mathrm{~km} \mathrm{~s}^{-1}\right.$, Howell et al. 1998). All the above values were, however, obtained from the radial velocity of the emission line wing, assumed equal to the white dwarf velocity. On the contrary, our results are the first to directly test the white dwarf absorption wings with the cross-correlation technique.

For $\mathrm{H} \alpha$ emission, we applied the "double Gaussian" convolution mask algorithm (Schneider \& Young 1980; Shafter 1983; Horne et al. 1986) which provides a robust diagnostic method to investigate the behavior of different profile sections during the orbital cycle. The method simultaneously shifts two Gaussians of standard deviation $\sigma_{\mathrm{g}}$ (or alternatively full width at half maximum $F W H M_{\mathrm{g}}$ ) and center separation $\Delta$ along the emission profile until a velocity is found for which the convolved flux in both is the same. Changing $\Delta$ and $F W H M_{\mathrm{g}}$ we can probe different velocity sections of every profile. This method is more versatile, allowing us to search for a better trade-off between noise and velocity resolution by changing $F W H M_{\mathrm{g}}$. It is superior to the simple Gaussian fit for the emission component.

The diagnostic diagram for the $\mathrm{H} \alpha$ emission in Fig. 6 shows the variations of $K$ and $\Delta \Phi$ (the phase shift with respect to the ephemeris defined by the absorption component) for velocity sets obtained with different Gaussian separations. We find that $K$ is large close to the line center but decreases towards the line wing, which could be the result of a systematic reduction of the $s$-wave perturbation toward the line wing. This is consistent with the diagnostic diagram starting with Gaussian separations close to the maximum $s$-wave velocity. In addition, the $\Delta \Phi$ behaviour indicates that the emission line center precedes the wing when crossing the zero phase. Interestingly, the emission line wing is almost in phase with the white dwarf motion. The above is consistent with wing emission arising from the inner disk and following more closely the white dwarf motion and low velocity emission coming from a hotspot in the disk-stream impact region close to the inner Lagrangian point. This view is also consistent with the fact that $K$ decreases towards the line wing (Fig. 6 upper panel), maybe approaching the white dwarf value, whereas the noise $\left(\sigma_{K} / K\right)$ becomes prominent.

Phase binned trailed spectra of the continuum and white dwarf subtracted spectra (see Sect. 3.5) are shown in Fig. 7. These spectra confirm the presence of an additional emission component. We find that this component is stronger in the higher-order Balmer lines and the He I 5875 line. This emission component is commonly associated with a hotspot in the disk and is called the " $s$ wave". Disk and hotspot are studied in the next section.

\subsection{Emission line analysis and the optical depth of the disk and the hotspot}

We measured the Balmer decrement for both the accretion disk and the hotspot region to check for different line opacities. The Balmer decrement $(D(\mathrm{H} \alpha / \mathrm{H} \beta))$ is defined
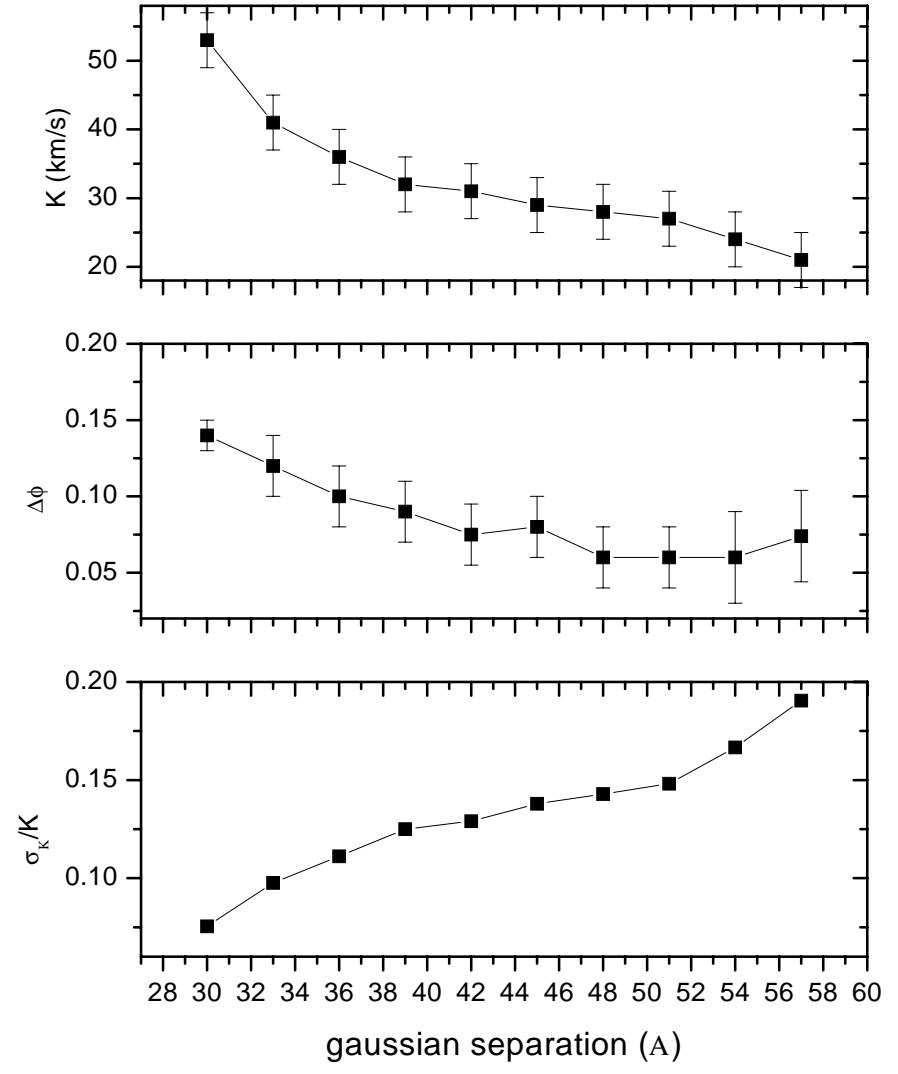

Fig. 6. The diagnostic diagram for the $\mathrm{H} \alpha$ emission line considering the ephemeris given by Eq. (1).

as the ratio between the frequency-integrated specific intensities of the $\mathrm{H} \alpha$ and $\mathrm{H} \beta$ lines. The fact that the disk and hotspot emission are simultaneously present in the line is a problem for the calculation of decrements. De-blending of hotspot and disk emission components is not possible without a complex physical model for the disk and hotspot regions. We find an approximate solution for this problem in the following way. We binned the observed spectra in 10 phase intervals and corrected them for the motion of the white dwarf. The white dwarf spectrum corresponding to the white dwarf parameters derived in Sect. 4 was subtracted from these spectra obtaining phase-binned emission profiles. To separate the emission components, we assumed symmetrical disk emission and separated the total profile for each bin in two components. The first consists mostly of disk emission, either the red or blue half of the profile depending on the $s$-wave velocity, and a second consisting of disk and hotspot emission. The larger and smaller peak had fluxes $f_{\max }$ and $f_{\min }$ respectively. The hotspot decrement was calculated for each phase-binned spectrum assuming that $f_{\text {hotspot }}=f_{\max }-f_{\min }$ and the disk decrement assuming $f_{\text {disk }}=f_{\text {min }}$. We also assumed a similar shape for the profiles at different wavelengths and applied a correction factor to calculate the decrements in the $f_{\lambda}$ space. Evidently the above is a rough approximation, and phase-bins when the profile was almost symmetric (i.e. the hotspot component was near the middle of the profile) were not measured. 


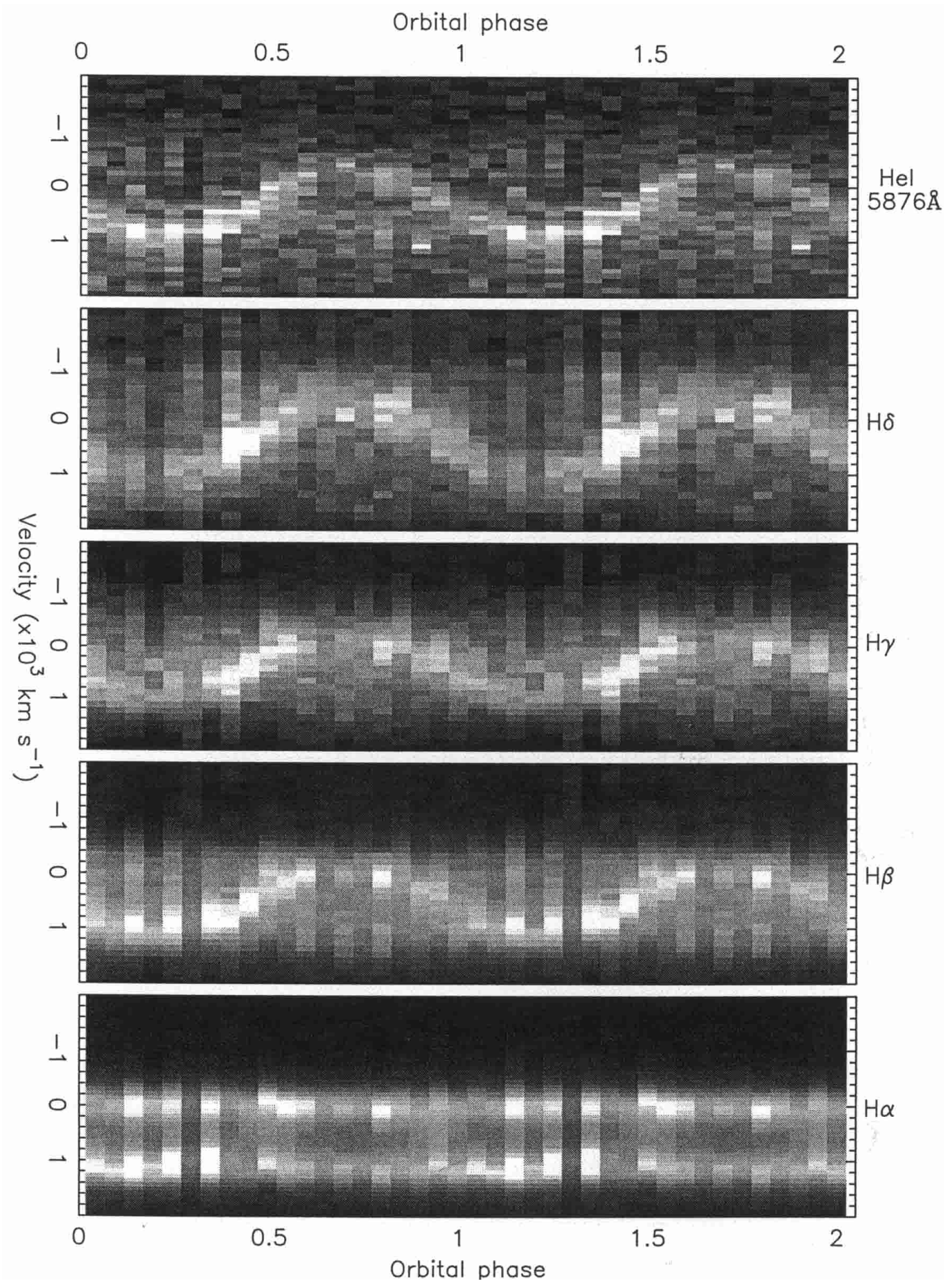

Fig. 7. The trailed spectra of the emission lines. The data have been binned into 20 phase bins and are repeated for clarity. Note how the relative strength of the bright spot emission with respect to the disk emission increases from $\mathrm{H} \alpha$ to $\mathrm{H} \delta$. A double peak is evident in $\mathrm{H} \alpha$.

Results and comparison with theoretical decrements by Williams (1991) are shown in Fig. 8. Williams modeled optically thin gas in the emission lines in accretion disks. He computed $\mathrm{H} \beta$ strengths and Balmer decrements for a grid of temperatures, inclinations and mid-plane accretion disk density $\left(N_{0}\right.$ in units of nucleons per $\left.\mathrm{cm}^{3}\right)$. Figure 8 shows the results of his models for a representative inclination of $52^{\circ}$ along with our observations. Results for different inclination angles are not significantly different. A direct interpretation of Fig. 8 is not possible since the decrements are not single-valued functions of disk temperature. However, considering some well-established facts, it is possible to extract some useful information. First, the observed disk decrement $(D(\mathrm{H} \alpha / \mathrm{H} \beta) \approx 2.3)$ indicates that for any realistic disk temperature $\left(T_{\text {disk }}>\right.$ few $\left.10^{3} \mathrm{~K}\right)$ the Balmer lines are optically thin. Second, the hotspot 


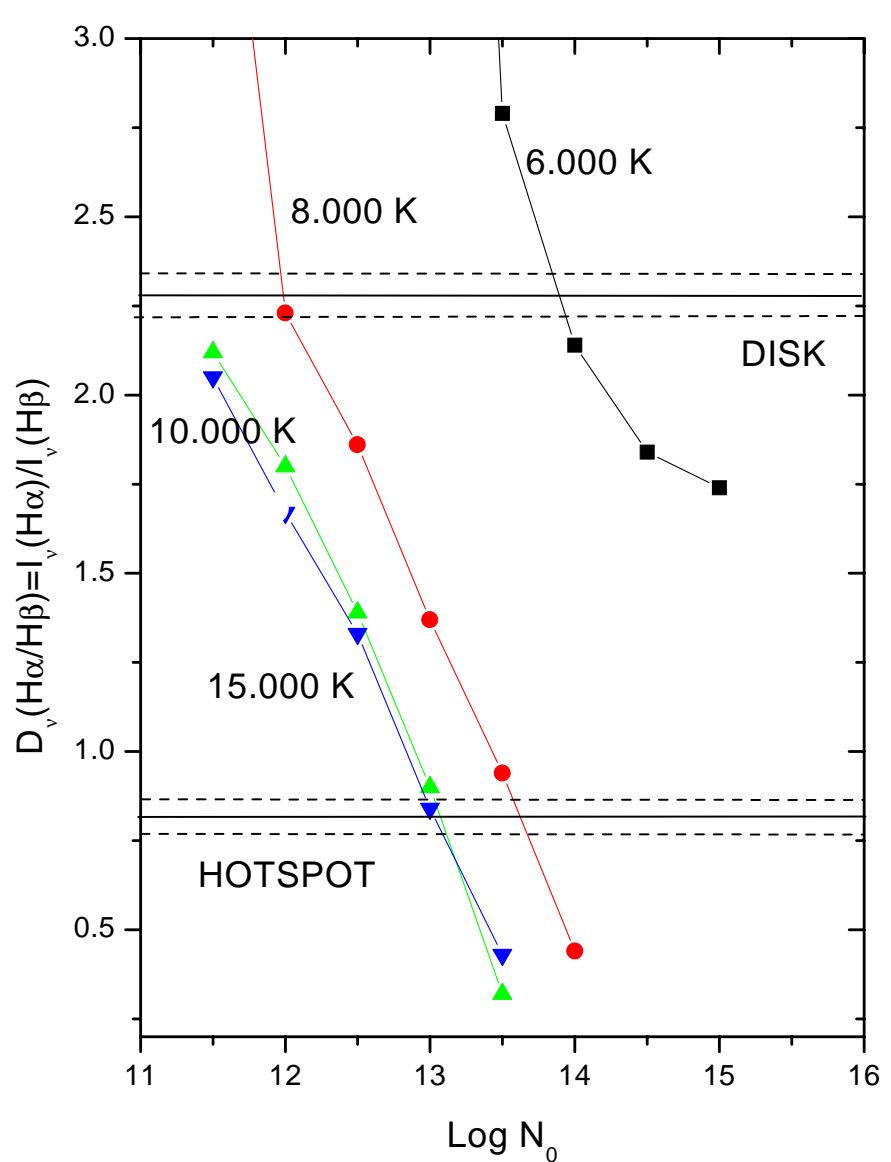

Fig. 8. Theoretical Balmer decrements for optically thin disks emission lines for inclination of $52^{\circ}$ according to Williams (1991). The models for $T=8000 \mathrm{~K}, 10000 \mathrm{~K}$, and $15000 \mathrm{~K}$, all approach to $D(\mathrm{H} \alpha / \mathrm{H} \beta)=1$ at high densities, as expected for optically thick lines. The lines indicate orbit-averaged decrements for disk and hotspot and their corresponding uncertainties.

Balmer decrement $(D(\mathrm{H} \alpha / \mathrm{H} \beta) \approx 0.8)$ is typical of optically thick line emission and, if the lines are saturated, it would correspond to a black-body temperature of $8000 \mathrm{~K}$. The difference between hotspot and disk decrement indicates a disk optically thinner than the hotspot in the lines. The previous analysis was applied to the prototype dwarf nova WZ Sge by Mason et al. (2000). These authors found values of 3.8 and 2 for the disk and hotspot decrements respectively, indicating that the disk density in 1RXS J105010.3-140431 is not as low as in WZ Sge.

\subsection{Evidence for inner disk removal}

In the previous section we have shown that the accretion disk of 1RXS J105010.3-140431 is similar in some regard to that found in long-cycle dwarf novae like WZ Sge. It has been suggested that these objects have disks which are depleted in their inner regions (e.g. Hameury et al. 2000; Mennickent 1999; Mennickent \& Arenas 1998). One method to test this scenario for 1RXS J105010.3-140431 is looking into the emission line widths. Smak (1981) found that the inner to outer disk radius ratio $R$ and the emissivity exponent $\alpha$ (assuming the emissivity $\propto r^{-\alpha}$ ) of an optical and geometrically thin Keplerian disk are functions of the emission line widths. For $\mathrm{H} \alpha$ we measured $1515,1915,2420 \mathrm{~km} \mathrm{~s}^{-1}$ at 80,40 and $10 \%$ of the maximum intensity level, respectively, yielding $R \approx 0.18$ and $\alpha \approx 1.5$. The emission line widths above were calculated after subtracting the underlying absorption profile. The corresponding figures for the raw emission are 0.22 and 1.6 respectively. We observe 1RXS J105010.3140431 close to WZ Sge in Fig. 8 by Mennickent \& Arenas (1998). The rather large $R$ value could indicate inner disk removal. Using Eq. (4) of Mennickent \& Arenas (1998) we calculated a supercycle length of about 5.2 years for 1RXS J105010.3-140431, which should explain the lack of observed outbursts. This recurrence time should correspond to a mass transfer rate $\dot{M} \sim 4 \times 10^{14} \mathrm{~g} \mathrm{~s}^{-1}$ according to Fig. 2 by Warner (1995). This is a very low mass transfer rate similar only to that of WZ Sge $\left(\dot{M} \sim 1 \times 10^{14} \mathrm{~g} \mathrm{~s}^{-1}\right)$ and HV Vir $\left(\dot{M} \sim 3 \times 10^{14} \mathrm{~g} \mathrm{~s}^{-1}\right.$, Warner 1995).

\subsection{Synthetic magnitudes and the upper limit for the systemic inclination}

Synthetic $V$ magnitudes were calculated from the fluxcalibrated spectra. Nightly mean values are given in Table 1. The seeing was stable during the four observing nights (less than 0.8 arcsec), therefore we are confident that the slit losses have little effect on the mean magnitudes, although they could be the cause of the larger rms compared with our photometric data. A plot of $V$ versus orbital phase does not reveal eclipses. This confirms the results of the differential photometry (Sect. 2.1).

We can get an upper limit for the inclination in a noneclipsing CV. For that we used the Eggleton (1983) approximation for the volume radius of the Roche lobe of the secondary star, obtaining:

$\tan \left(\frac{\pi}{2}-i_{\max }\right)=\frac{0.49 q^{2 / 3}}{\left(1-r_{\mathrm{d}} / a\right)\left[0.6 q^{2 / 3}+\ln \left(1+q^{1 / 3}\right)\right]}$

where $q=\frac{M_{2}}{M_{1}}$ is the mass ratio and $a$ the binary separation. Using the approximation $r_{\mathrm{d}} / a=0.6 /(1+q)$ (Warner 1995 , Eq. (2.61)), an upper limit $i_{\max } \approx 65^{\circ}$ is found almost independent of $q$.

\subsection{Constraints on the stellar masses}

The evolution of cataclysmic variables has been recently reviewed with the use of a population synthesis code by Howell et al. (2001). The requirement of a Roche lobefilling secondary allows tracing of the evolution of the secondary star mass as a function of the orbital period. For a given orbital period, two solutions are possible, depending on whether the system has $\dot{P}<0$ or $\dot{P}>0$. From Fig. 5 of Howell et al. (2001) and using $P=88.6 \mathrm{~min}$ we obtain $M_{2}=0.14 M_{\odot}$ and $M_{2}=0.05 M_{\odot}$ respectively. The first solution corresponds to a system with a nearly main-sequence secondary burning hydrogen in its 
core. The system moves to shorter orbital periods as it loses angular momentum by gravitational radiation. The second solution corresponds to a system with a secondary star having a hydrogen-exhausted degenerated core, like a brown dwarf star, and moving to longer orbital periods after "bouncing" in the orbital period minimum around $80 \mathrm{~min}$. In order to discriminate between both possible scenarios, it is necessary to use a set of empirical constraints, which we discuss below.

The condition that the $H W Z I$ of the emission lines cannot be greater than the Keplerian velocity corresponding to the primary radius, combined with the white dwarf mass-radius relation by Hamada \& Salpeter (1969), yields $M_{1}>0.32 M_{\odot}$. For this calculation we used the He 5875 line width $\left(F W Z I=3680 \mathrm{~km} \mathrm{~s}^{-1}\right)$, that probably is not influenced by underlying absorption.

The low $K_{\mathrm{wd}}$ also provides strong constraints on the stellar masses and systemic inclination through the mass function:

$2 \pi G M_{1} \sin ^{3} i=P_{\mathrm{o}} K_{\mathrm{wd}}^{3} \frac{(1+q)^{2}}{q^{3}}$.

All the above constraints are plotted in Fig. 9. It is evident that a main sequence secondary is incompatible with the low $K_{\mathrm{wd}}$ value. The formal solution gives a secondary mass between 10-20 Jovian masses for inclination angles between 65 and 35 degree. The inclination would have to be below 5 degrees for a main sequence companion, which is highly unlikely due to the $F W Z I$ of $\mathrm{He} \mathrm{I}$ and the $s$-wave amplitude. Even if for some unknown reason the absorption lines do not follow the white dwarf motion, but the emission wing velocity tends to this value, the mass function for $K=20 \mathrm{~km} \mathrm{~s}^{-1}$ implies that the inclination should be lower than 25 degree for a hydrogen burning companion, which is possible but unlikely. The mass transfer rate derived in Sect. 3.4 , viz. $6.3 \times 10^{-12} M_{\odot} \mathrm{yr}^{-1}$, and the low mass ratio $(q \leq 0.09$ derived from the empirical relationship by Mennickent (1999) using the $K_{\mathrm{wd}} / F W H M$ ratio) nicely fit the prediction of Howell et al. (2001) for a post-minimum system with orbital period around $89 \mathrm{~min}$.

We have found the first dynamical evidence for a cataclysmic variable beyond the orbital period minimum. The first objects belonging to this class, viz. LL And and EF Eri, have recently been discovered by detecting the secondary features in the infrared by Howell \& Ciardi (2001), although indirect evidence has been provided for other objects previously, namely AL Com (Howell et al. 1998), WZ Sge (Ciardi et al. 1998), V592 Her (van Teeseling et al. 1999) and EF Eri (Beuermann et al. 2000).

\subsection{Imaging the accretion disk with Doppler tomography}

The spectroscopic data were phased according to the orbital ephemeris given above. The continuum was subtracted off in two stages. First, a cubic polynomial was fit to the continuum regions between the white dwarf absorption dips and subtracted. Second, a $15000 \mathrm{~K}$ Pop II white

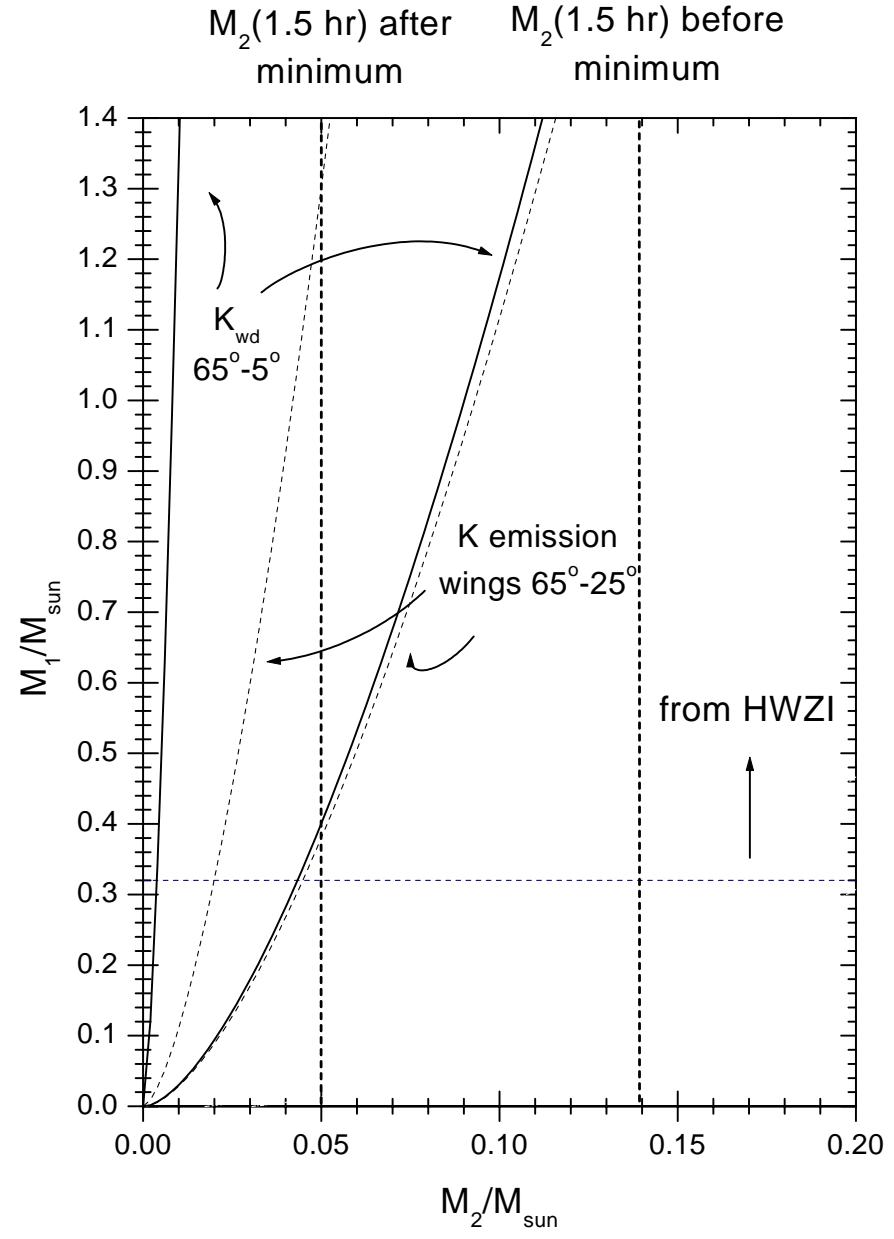

Fig. 9. The $M_{1}-M_{2}$ plane showing the dynamic solutions for $K_{1}=4 \mathrm{~km} \mathrm{~s}^{-1}$ (absorption wings) and $K_{1}=20 \mathrm{~km} \mathrm{~s}^{-1}$ (emission wings). The vertical lines indicate possible secondary star masses for the orbital period accordingly to results of the evolutionary code by Howell et al. (2001). A post orbital period minimum secondary is favoured by our velocities.

dwarf model, convoluted with the instrumental resolution, and with the continuum removed using a polynomial as above, was fit to the white dwarf absorption dips in the data and subtracted to leave only the line emission. Using maximum entropy Doppler tomography (Marsh \& Horne 1988) we constructed Doppler maps of the $\mathrm{H} \alpha, \mathrm{H} \beta, \mathrm{H} \gamma, \mathrm{H} \delta$ and He I 5876 lines. The data were not phase-binned before constructing the Doppler maps; this maximized phase coverage with 57 spectra covering all orbital phases observed. The maps are shown in Fig. 10. The $\mathrm{H} \alpha$ map shows a symmetric disk and a weak hotspot with velocities between that of the ballistic stream and local Keplerian velocity. In the $\mathrm{H} \beta, \mathrm{H} \gamma, \mathrm{H} \delta$ maps the disk becomes progressively fainter, while the hotspot becomes stronger and shifts to velocities closer to the local Keplerian velocity. This is consistent with high order Balmer lines formed deeper in the ballistic stream, where the excess of kinetic energy is radiated by interaction with the disk. The He I 5876 map shows low velocity bright spot emission; this may indicate substantial photoionization of the gas stream 

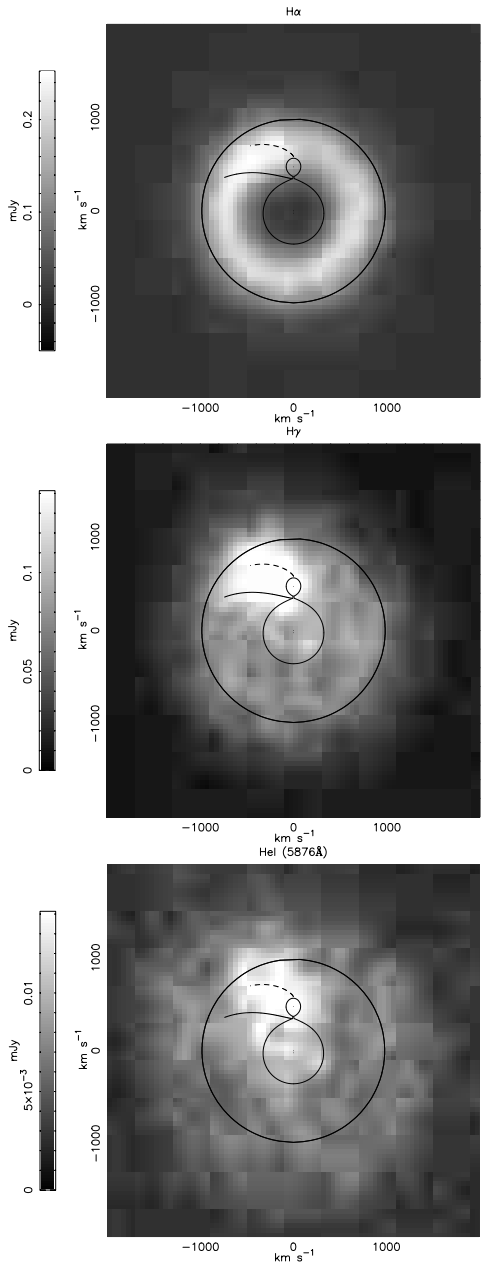
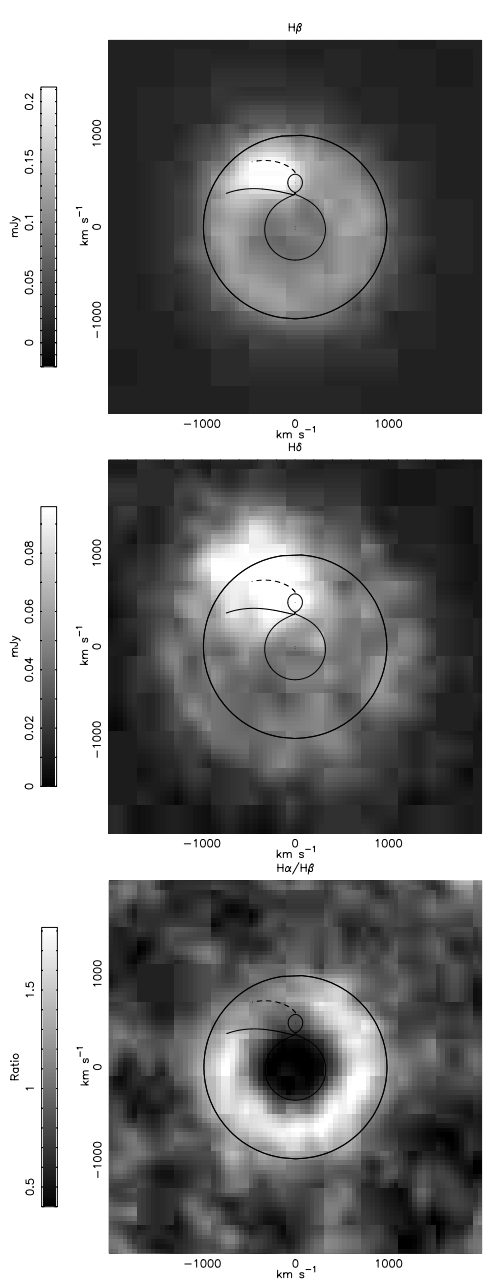

Fig. 10. Doppler maps of the $\mathrm{H} \alpha, \mathrm{H} \beta, \mathrm{H} \gamma, \mathrm{H} \delta$ and $\mathrm{He}$ I 5876 emission lines. Overlayed is the Roche geometry of the system with $M_{1}=1.0 M_{\text {sun }}, M_{2}=0.05 M_{\text {sun }}, P_{\text {orb }}=0.0615 \mathrm{~d}$ and $i=65 \mathrm{deg}$. The dashed line shows the local Keplerian velocity along the stream path assuming circular orbits around the primary. The circular line represents $1 / 3$ of the primary Roche lobe radius. The relative increase in bright spot strength with respect to the disk emission is evident as we go from $\mathrm{H} \alpha$ to $\mathrm{H} \delta$. Note that the He I bright spot is positioned with a slower velocity than the H I bright spots. In general the bright spots have velocity between that of ballistic stream and the local Kepler velocity. Bottom right: the Ratio map of the $\mathrm{H} \alpha$ and $\mathrm{H} \beta$ Doppler maps. Note the striking similarity in the structure of the ratio map of 1RXS J105010.3-140431 to that of WZ Sge shown in Fig. 6 of Skidmore et al. (2000).

and/or that the He I bright spot originates further up the ballistic stream than the H I bright spot, suggesting that the accretion disk extends further out than would be deduced from the $\mathrm{H}$ I emission alone. In the nova-like system V347 Pup for instance, the He II emission is produced at inner parts of the disk when compared with H I (Diaz \& Hubeny 1999).

The Ratioed Doppler Map for 1RXS J105010.3-140431 is shown in the bottom right of Fig. 10; this was constructed by dividing the $\mathrm{H} \alpha$ map by the $\mathrm{H} \beta$ map shown in Fig. 10. The change in the Balmer decrement in the region of the bright spot indicates hotter optically-thick emission than is seen from the disk. It is interesting to note the similarity in the structure of the ratioed Doppler maps of 1RXS J105010.3-140431 and that of WZ Sge (Skidmore et al. 2000). However, the ratios of the disk and hot spot emission measured in 1RXS J105010.3-140431 are generally lower than measured in WZ Sge, indicating higher temperatures and/or greater optical depth in 1RXS J105010.3-140431, as found in Sect. 3.3.

\subsection{Radial brightness temperature profile}

Using the same method as described in Skidmore et al. (2000), the radial profile of the ratioed Doppler map was calculated by finding the average ratio at each radius in velocity space. Areas of the ratio map affected by the bright spot flux were masked when calculating the radial profile. These areas covered between -20 and 75 degrees measured anti-clockwise from the $y$ axis. The mean line ratio at each velocity was used to determine a temperature assuming a simple blackbody source function at the wavelength of the line emission. This oversimplification is used as a rough guide to infer temperature gradients in the disk. The system parameters for 1RXS J105010.3140431 determined in this paper were used to convert the 


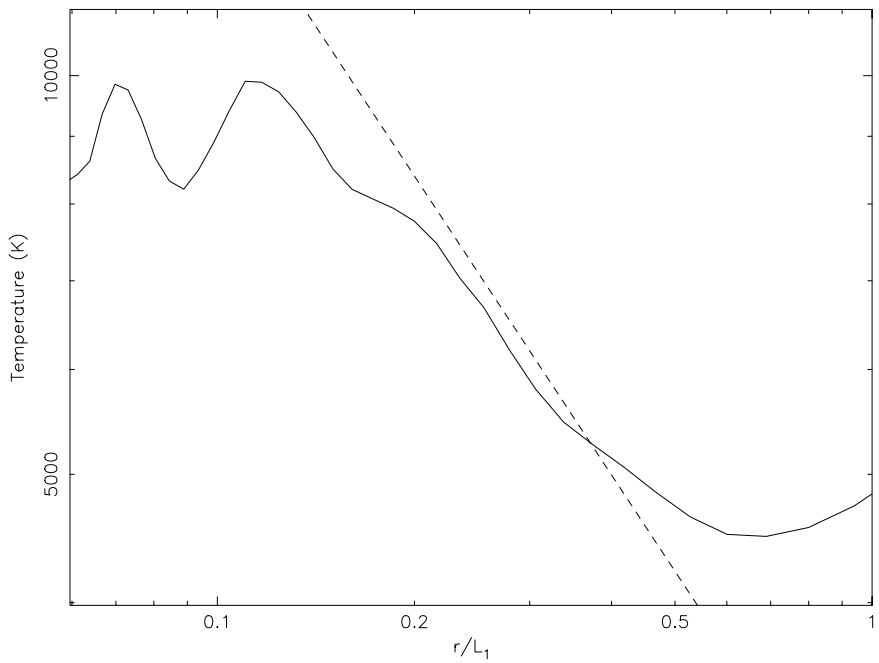

Fig. 11. The radial temperature profile for the disk emission in 1RXS J105010.3-140431 assuming a blackbody source function. The diagonal dashed line indicates the slope of the radial temperature profile for a standard alpha disk where $T \sim r^{-3 / 4}$.

velocity space radial temperature profile into a real space radial temperature profile. The radial temperature profile for 1RXS J105010.3-140431 is shown in Fig. 11.

We note that the inner disk temperatures are larger than those suggested by the simple decrement analysis. Apparently the decrement study gives more weight to the outer disk regions. This is easily explained since there is a larger contribution of these low velocity regions to the emission profile and the decrement study is based on the integrated flux along the profile.

As the slope of the radial temperature in the disk is close to $T \sim r^{-3 / 4}$, we can see that the disk is in a steady state. In quiescent dwarf novae the disk radial temperature profile is usually observed to depart from the $r^{-3 / 4}$ law and to have a much flatter profile (Wood et al. 1992; Skidmore et al. 2000). The departure from $T \sim r^{-3 / 4}$ occurs at radii of $r_{\text {in }} \sim 0.1$ and $r_{\text {out }} \sim 0.5$. If these radii represent the inner and outer edges of the disk then $R=\frac{r_{\text {in }}}{r_{\text {out }}} \sim 0.2$ as found in Sect. 3.4.

\section{Modeling the absorption spectrum}

The observed spectrum of 1RXS J105010.3-140431 clearly shows broad Balmer absorption profiles flanking the emission lines. These features present extended line wings typical of Stark broadened Balmer lines; therefore, we may associate them with the underlying white dwarf spectrum, instead of Doppler-broadened absorption from the accretion disk photosphere. The conspicuous presence of the Balmer absorption component indicates that the white dwarf photosphere is bright when compared to the accretion disk continuum. In most accreting cataclysmic variable systems the accretion disk flux prevents the observation of the compact star. The detection of the white dwarf in 1RXS J105010.3-140431 offers the opportunity of obtaining basic photospheric parameters for the white dwarf by comparing the observed profiles with a grid of synthetic spectra. On the other hand, helium absorption features could not be identified in the profiles of He I $\lambda \lambda 6678,5876,4471 \AA$. This fact suggests a DA classification for the white dwarf.

\subsection{High-gravity atmosphere models and spectrum synthesis}

In order to obtain a grid of model DA white dwarf spectra, pure hydrogen atmospheres were calculated assuming hydrostatic and radiative equilibrium. Only the continuum and hydrogen opacity are included, along with scattering and free particle processes. The plane-parallel atmosphere structure is solved locally at 70 mass-depth points by the complete linearization method using the code TLUSTY (Hubeny \& Lanz 1995).

Once the density, temperature and opacity is calculated, the radiative transfer equation is solved for each frequency in the region of interest by the program SYNSPEC (Hubeny et al. 1994). NLTE departures are allowed for the hydrogen levels population. The improved calculations of the Stark broadening by Shoning \& Butler (1989) in addition to the thermal Doppler component are used to compute the Balmer line profiles. Both emergent flux $\left(\mathrm{H}_{\lambda}\right)$ and theoretical continuum are computed in absolute units. Our line profile calculations were found to be in good agreement with the white dwarf models by Jordan \& Koester (1986).

\subsection{Model fitting and white dwarf parameters}

About 90 model spectra were computed with effective temperatures ranging from $10000 \mathrm{~K}$ to $65000 \mathrm{~K}$ and $\log g$ between 6.0 and 9.5. Continuum subtracted and continuum normalized model sets were prepared using the theoretical continuum calculated in the absence of boundbound transitions. Subsequently we fitted a low order polynomial pseudo-continuum to the models in the region covered by our spectroscopy. This residual continuum correction of model spectra is necessary for their comparison with the observations because of the significant overlapping of the Balmer line wings beyond $\mathrm{H} \beta$.

The regions for fitting were carefully selected in the observed spectrum to exclude the contribution from the narrow central emission and from weak emission lines in the extended wings. Two different procedures were employed to fit model spectra to the observations:

The first method consists of fitting the line profile in flux units using continuum-subtracted spectra. Each model spectrum in the grid is arbitrarily scaled to match the integrated flux in the observed absorption lines and then the reduced chi-square statistic is computed for each pair $\left(T_{\text {eff }}, \log g\right)$. This method does not require a previous knowledge of accretion disk continuum contribution to the total flux. However, it has the disadvantage of disregarding the line to continuum flux ratio. Results from 


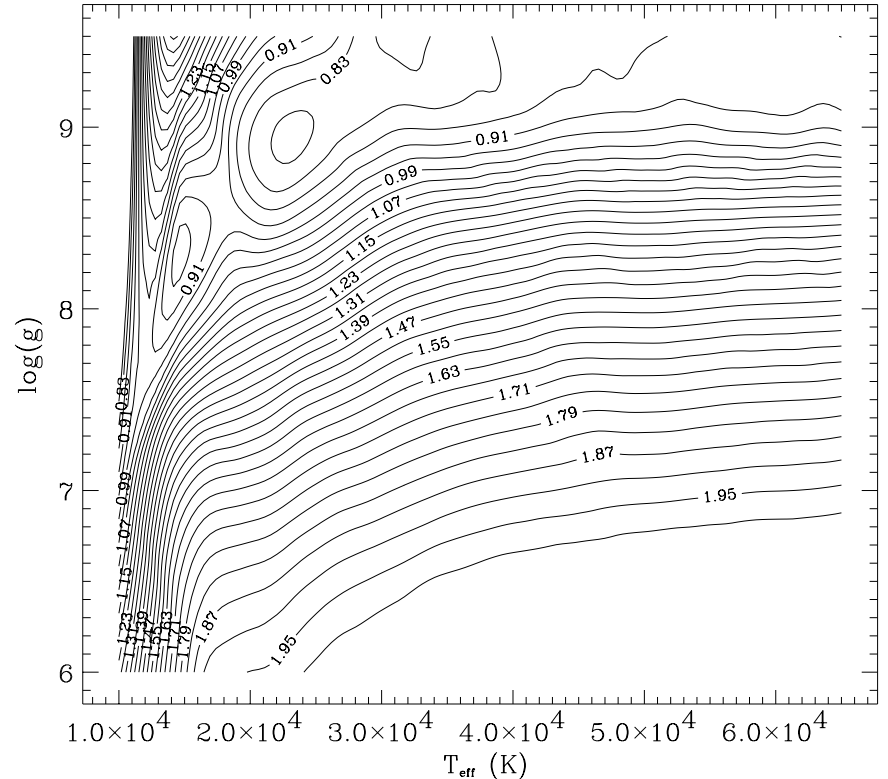

Fig. 12. $\chi^{2}$ as a function of surface gravity and temperature according to the results of method 1 .

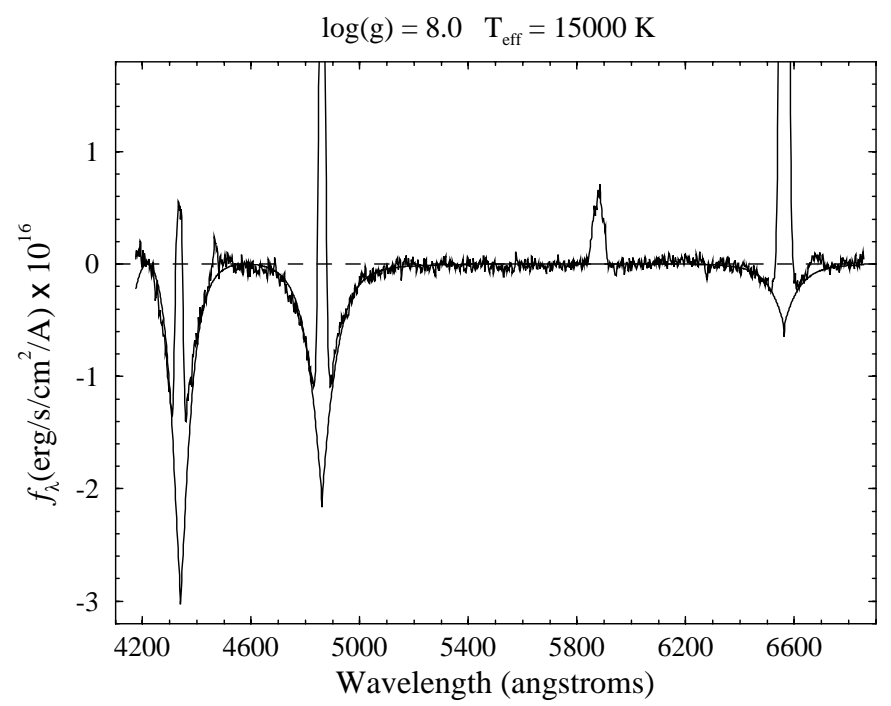

Fig. 13. Illustration of the spectrum synthesis analysis.

this method are shown in Fig. 12. A valley in the $\chi^{2}$ surface is evident from the graph, containing two minima. A sample individual fit can be seen in Fig. 13.

A caveat related to the effect of the unknown interstellar extinction should be made here. This effect, in principle, must be considered when the simultaneous fitting of $\mathrm{H} \alpha, \mathrm{H} \beta$ and $\mathrm{H} \gamma$ is performed. However, a comparison with the fit of $\mathrm{H} \beta$ and $\mathrm{H} \gamma$ alone does not show significant discrepancies. In addition, the expected extinction at the target galactic latitude $\left(b_{\mathrm{II}}=39 \mathrm{deg}\right)$ should be low.

The second method uses the continuum-normalized spectrum as input and deals with the veiling by the other continuum sources in the binary. Unfortunately, the shape of the underlying disk continuum emission is unknown. However, given its low luminosity relative to the white dwarf one may expect under standard theory that such a

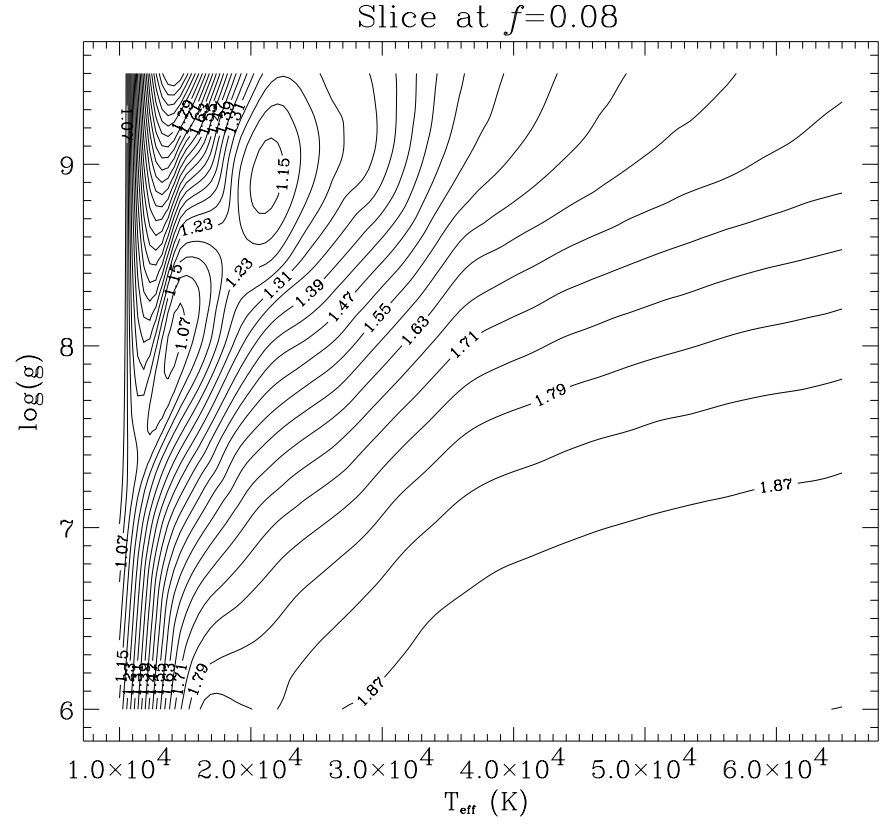

Fig. 14. $\chi^{2}$ as a function of surface gravity and temperature according to the results of method 2. The plot corresponds to a value of $f=0.08$ (see text).

disk should present relatively low temperatures. For the sake of simplicity it was assumed that the disk continuum shape is similar to the white dwarf continuum, but scaled by an unknown factor. The relative disk contribution to the total flux $(f)$ is independent of the wavelength and may be left as a free parameter in the model fitting. Typical results are shown in Fig. 14, where the behavior is similar to that seen in Fig. 12. Simulations show that the minima drift toward lower temperatures and higher gravities as the veiling factor $f$ increases. The increase in $f$ also implies a significant decrease in the quality of the fit. It became clear from our simulations that the disk should contribute less than $20 \%$ of the system flux.

The dynamical solution discussed in Sect. 3.6 gives a range for the white dwarf mass too large to discriminate between the two minima found in the $\chi^{2}$ plots. By combining the quality criteria obtained in both procedures we estimate $\log g$ ranging from 7.6 to 9.3 and $T_{\text {eff }}$ from $13000 \mathrm{~K}$ to $24000 \mathrm{~K}$.

\section{Conclusions}

- A spectroscopic analysis of the ROSAT target 1RXS J105010.3-140431 has been realized noting the close similarity between this source and the prototype dwarf nova WZ Sge. In particular, the white dwarf photospheric lines are revealed in the spectrum, which is a signature of a very low mass accretion rate.

- We find a most likely orbital period of $88.6 \mathrm{~min}$ although \pm 1 cycle/day aliases cannot be ruled out.

- The absorption spectrum can be modeled with a DA white dwarf with $13000 \mathrm{~K}<T_{\text {eff }}<24000 \mathrm{~K}$. 
- The mass function suggests that the system is beyond the orbital period minimum having an undermassive secondary.

- As in other short orbital period dwarf novae systems, evidence for inner disk removal is found. In addition, using empirical relationships for dwarf novae, we predict a supercycle of several years for this object.

- The Doppler maps and the Balmer decrement study reveal significant differences between the optical depth of the disk and hotspot. We find an optically thick hotspot with $T \sim 8000 \mathrm{~K}$ and an optically thinner disk. The hotspot has velocities consistent with dissipation of kinetic energy in the stream-disk interacting region, and it is the main emitting source in high order Balmer lines and the He I 5875, but not in $\mathrm{H} \alpha$, where the emission is mainly distributed along a symmetrical disk. Contrary to the Balmer lines, the helium line seems to be formed in the outer stream-disk impact region.

- We constructed the temperature profile for the disk and, contrary to that found in other dwarf novae, we find an almost steady state standard disk, with $T \sim r^{-3 / 4}$.

Acknowledgements. This work was partly supported by Fondecyt 1000324, D.I. UdeC 99.11.28-1 and Fundacion Andes C-13600/5. This project was also supported by the Flemish Ministry for Foreign Policy, European Affairs, Science and Technology and by FAPESP 9906261 and CNPq 301029. We thank T. Augusteijn for calling our attention to this interesting object during an observing session in the La Silla Observatory. We also thank T. Marsh for the use of his MOLLY spectral analysis software and DOPPLER maximum entropy Doppler tomography software. We thank D. Koester for providing the white dwarf spectrum used during the production of the Doppler maps. We also thank L. Barros for her kind help during the photometric reductions.

\section{References}

Beuermann, K., Wheatly, P., Ramsey, G., Euchner, F., \& Gansicke, B. 2000, A\&A, 354, L49

Ciardi, D., Howell, S. B., Hauschildt, P., \& Allard, F. 1998, ApJ, 504, 450

Diaz, M. P., \& Hubeny, I. 1999, ApJ, 523, 786

Eggleton, P. P. 1983, ApJ, 268, 368
Friend, M. T., Martin, J. S., Connon-Smith, R., \& Jones, D. H. P. 1990, MNRAS, 246, 654

Hamada, T., \& Salpeter, E. E. 1961, ApJ, 134, 683

Hameury, J., Lasota, J., \& Warner, B. 2000, A\&A, 353, 244

Hamuy, M., Walker, A. R., Suntzeff, N. B., et al. 1992, PASP, 104,533

Hamuy, M., Suntzeff, N. B., Heathcote, S. R., et al. 1994, PASP, 106, 566

Hessman, F. V. 1988, A\&AS, 72, 515

Howell, S. B., \& Ciardi, D. R. 2001, ApJ, 550 L57

Howell, S. B., Wamock, III A., \& Mitchell, K. J. 1988, AJ, 95, 247

Howell, S. B., Hauschildt, P., \& Dhillon, V. S. 1998, ApJ, 494, L223

Howell, S. B., Nelson, L. A., \& Rappaport, S. 2001, ApJ, 550, 897

Hubeny, I., Lanz, T., \& Jefferys, C. S. 1994, CCP7 Newslett.

Hubeny, I., \& Lanz, T. 1995, ApJ, 439, 875

Horne, K., Wade, R. A., \& Szkody, P. 1986, MNRAS, 219, 791

Jordan, S., \& Koester, S. 1986, A\&AS, 65, 367

Marsh, T., \& Horne, K. 1988, MNRAS, 235, 269

Mason, E., Skidmore, W., Howell, S., et al. 2000, MNRAS, 318, 440

Mennickent, R. E. 1999, A\&A, 348, 364

Mennickent, R. E., \& Arenas, J. 1998, PASJ, 50, 333

Robinson, E. L. 1992, in Vina del Mar Workshop on Cataclysmic Variable Stars, ed. N. Vogt, ASP Conf. Ser., 29,3

Scargle, J. D. 1982, ApJ, 263, 835

Schneider, D. P., \& Young, P. 1980, ApJ, 238, 946

Schwarzenberg-Czerny, A. 1989, MNRAS, 241, 153

Shafter, A. W. 1983, ApJ, 267, 222

Shoning, T., \& Butler, K. 1989, A\&AS, 78, 51

Skidmore, W., Mason, E., Howell, S., et al. 2000, MNRAS, 318, 429

Smak, J. 1981, Acta Astron., 31, 395

Smith, D. A., \& Dhillon, V. S. 1998, MNRAS, 301, 767

Spruit, H. C., \& Rutten, R. G. M. 1998, MNRAS 299, 768

Szkody, P., Desai, V., \& Hoard, D. W. 2000, ApJ, 119, 365

Tonry, J., \& Davis, M. 1979, AJ, 84, 1511

van Teeseling, A., Hessman, F., \& Romani, R. 1999, A\&A, 342, L45

Voges, W., et al. 1996, IAU Circ., 6420

Warner, B. 2000, PASP, 112, 1523

Warner, B. 1995, Cataclysmic Variable Stars (Cambridge University Press)

Williams, G. 1991, AJ, 101, 1929

Wood, J., Horne, K., \& Vennes, S. 1992, ApJ, 385, 294 